\title{
A Regressive Problem Solver That Uses Knowledgelet
}

\author{
Kuodi Jian \\ Department of Information and Computer Science \\ Metropolitan State University \\ Saint Paul, Minnesota 55106-5000 \\ Kuodi.jian@metrostate.edu
}

\begin{abstract}
This paper presents a new idea of how to reduce search space by a general problem solver. The general problem solver, Regressive Total Order Planner with Knowledgelet (RTOPKLT), can be used in intelligent systems or in software agent architectures. Problem solving involves finding a sequence of available actions (operators) that can transfer an initial state to a goal state. Here, a problem is defined in terms of a set of logical assertions that define an initial state and a goal state. With too little information, reasoning and learning systems cannot perform in most cases. On the other hand, too much information can cause the performance of a problem solver to degrade, in both accuracy and efficiency. Therefore, it is important to determine what information is relevant and to organize this information in an easy to retrieve manner.
\end{abstract}

Keywords: Software Agent, Artificial Intelligence, Planning.

\section{Introduction}

In recent years, as the computing power increases steadily, computers are used in a wide variety of areas that need some intelligence. All of these intelligent systems need a problem solver. The problems that require a problem solver include: (1) generating a plan of action for a robot, (2) interpreting an utterance by reasoning about the goals a speaker is trying to achieve, (3) allocating the use of resources, and (4) automatically writing a program to solve a problem. The heart of a problem solver is a planner. There are many kinds of planners [1]. In this paper, we present a regressive planner that uses knowledgelet. A planner is complete if it is able to find every solution given that solutions exist and that the planner runs to its completion. There is a trade-offs between the response time and the completeness. We can improve the response time of a planner by using some criteria to prune the search space. But that will cause the loss of solutions to the problem. On the other hand, if a planner searches every possible space, then, the search space grows exponentially with the number of operators; the response time will deteriorate to the point of intolerable for many problems of decent size. This paper offers a solution that gives us both the quick response time and the completeness of solutions. The planner presented in this paper has the following characteristics: 
- It has fast response time since the planner searches only relevant operators,

- It can use existing relational database to store pieces of knowledge, called knowledgelet,

- It is built on proven regressive idea and is conceptually simple and complete,

- It is general, which means that it can be used in different areas with little or no changes.

The rest of the paper is organized as the following: section 2 gives the description of knowledgelet; section 3 presents the regressive general problem solver, RTOPKLT, and its application to a planning problem; and section 4 is the summary.

\section{Knowledgelet}

A knowledgelet is an object that describes the relevant knowledge of solving a particular problem. By using knowledgelets, the search time by a problem solver can be greatly reduced. A knowledgelet consists of slots of data structure and can be stored in most existing databases (object databases will be the best fit).

\subsection{Structure of Knowledgelet}

Knowledgelet constrains the world states by giving only subsets of operators (actions) that are relevant to the problem at hand. The search space can be an open world space or a Local Closed World (LCW) space [2]. In a LCW space, we assume that we know

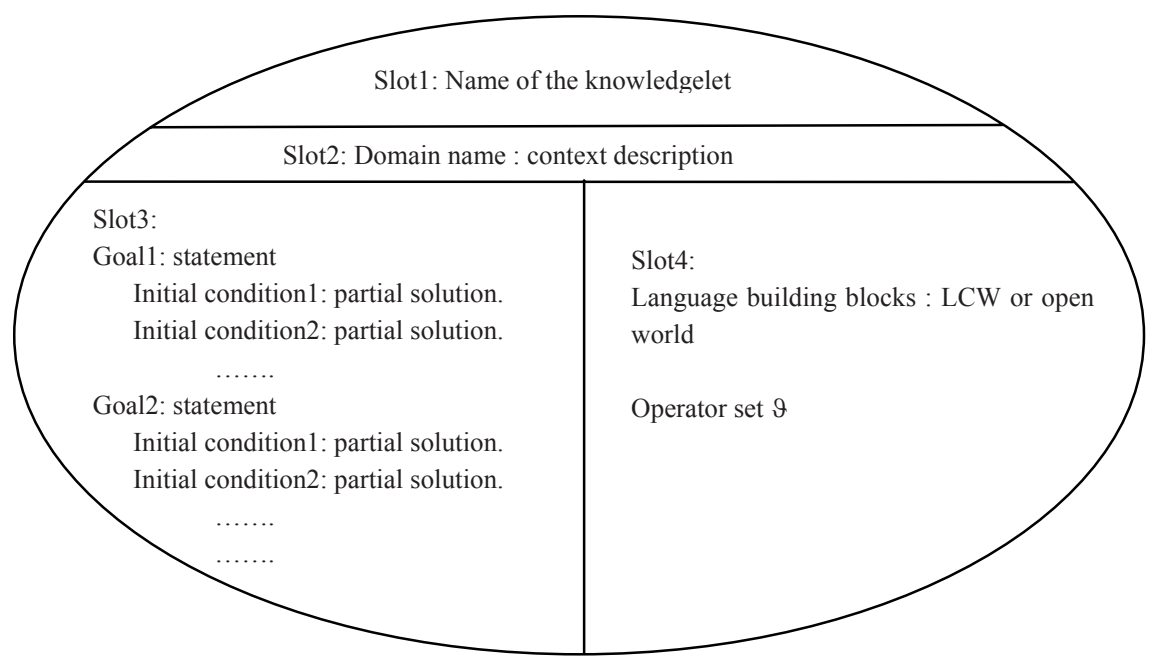

Fig. 1. The structure of a knowledgelet 
everything. If something is not positively stated, we conclude it is false. The benefit of using LCW is that we needn't to record all the world states. Figure 1 is the diagram of a knowledgelet.

From the diagram, we see that a knowledgelet has a name. This name can be used by a database as a key to store it. A knowledgelet is like a blob of knowledge with a domain name (also can be used as a key) and a context that describes the domain name. We all know the fact that the same words carry different meanings in different contexts. The domain name and its context will solve this ambiguity.

In slot3, there are goals that can be searched. Under each goal, there is a set of partial solutions to the goal. Each partial solution is associated with an initial state. This means that the same goal can be achieved from different starting points. For example, in a block world, the goal of block $\mathrm{C}$ on top of block $\mathrm{B}$ that is in turn on top of block A can be achieved either from the initial state of (on A B) ^ (on C Table) or from the initial sate of $($ on A Table $) \wedge($ on B Table $) \wedge($ on $C$ Table $)$. A partial solution is a partial plan (contains an ordered sequence of operators) that will change the world from an initial state to the goal state. To the regressive planner, this partial plan can be viewed as one big operator [3].

In slot 4 , there is a field that contains the language type and a field that contains available operator set. There are two language types: open world assumption and the Local Closed World (LCW) assumption. The LCW language building block is a LCW lingo in that particular domain; the open world language building block is an open world lingo in that particular domain. The language building blocks include sentences, axioms, rules, and constraints. One example of the constraints is the domain rules. Domain rules are the guiding principles that operate in a particular domain. They may specify a particular sequence of operators (for example, one operator must proceed another, etc.). If there is no existing partial plan in slot3 to match a goal, the planner will construct a plan from the available operators in slot4 with the help of the language lingo in the language type field.

The search starts from slot1, slot2, slot3, and slot4. By searching the partial solution first, we improve the response time since we do not have to construct everything from scratch.

\section{Regressive General Total Order Planner, RTOP}

In artificial intelligence, problem solving involves finding a sequence of actions (operators) that result in a goal state. A lot of real world problems can be cast into planning problems in terms of an initial state, a set of goal state conditions, and a set of relevant legal operators; each legal operator is defined in terms of preconditions and effects. Preconditions of an operator must be satisfied before the operator can be applied. All the reachable states comprise world space. A world state in a world space can be represented by state variables and can only be changed by legal operators.

There are basically two ways to search through a world space. One is called "progression" and the other is called "regression." A progressive planner searches 
forward from the initial world state until it finds the goal state. A regressive planner searches backward from the goal state until it finds the initial state. As the search progresses, both the forward search algorithm and the backward search algorithm need to choose non-deterministically the next operator to consider. The planner discussed in this paper belongs to the regressive type. Since the answer returned by our planner is a total-ordered (represented by " $<<$ ") sequence of operators, our planner is called Regressive Total Order Planner with Knowledgelet (RTOPKLT).

In this planner, a returned plan is represented by two-tuple: $<A$, TOLO $>$. TOLO is the total order of the operators that is selected by the planner. Internally, a plan is represented by a three-tuple: $<\mathrm{A}, \mathrm{O}, \mathrm{L}>$. A is a set of operators drawn from available operator pool $\vartheta, \mathrm{O}$ is a set of partial-ordering (represented by " $<$ ") constraints over A, and $L$ is a set of causal links. For example, if $A=\left\{A_{1}, A_{2}, A_{3}\right\}$ then $O$ might be the set $\left\{A_{1}<A_{3}, A_{2}<A_{3}\right\}$. A good way of ensuring that the different operators introduced for different goals won't interfere with each other is to record the dependencies among operators explicitly within a partial plan. To record these dependencies, we use a data structure called causal link [4]. A causal link is a data structure with three fields: the first two fields contain pointers to plan operators (the link's producer, $A_{p}$, and its consumer, $A_{c}$ ); the third field is a proposition or conjunct of propositions, $Q$, which is both an effect of $A_{p}$ and a precondition of $A_{c}$. We can write such a causal link as $A_{p} \rightarrow(Q) A_{c}$ and store a plan's links in the set L. Figure 2 is the algorithm for the regressive planner.

\section{Algorithm: RTOPKLT}

$<$ A, TOLO>RTOPKLT_Algorithm (InitialState, Goal, KnowledgeletName, DomainName)

(a) Use either KnowledgeletName or DomainName to search existing knowledgelets.

If there is a applicable knowledgelet in the system and the partial plan exist

for the given InitialState and Goal

Return the plan directly

Else if there is a applicable knowledgelet in the system and the partial plan does not exist for the given IntialState and Goal

1. Construct $\mathrm{A}_{0}, \mathrm{~A}_{\infty}$, initial $\mathrm{A}, \mathrm{O}, \mathrm{L}$, agenda, and get $\vartheta$ from knowledgelet. All the added operator $\mathrm{A}_{i}$ satisfies the following ordering:

$$
\mathrm{A}_{0}<\mathrm{A}_{\mathrm{i}}<\mathrm{A}_{\infty}
$$

2. Goto step (b), pass it a null plan, initial agenda, and the operator pool $\vartheta$.

Else if there is no applicable knowledgelet in the system

Return no answer.

(b) Call subroutine $\mathrm{RTOP}(<\mathrm{A}, \mathrm{O}, \mathrm{L}>$, agenda, ९).

(c) Total order: TOLO $=$ total ordering $\mathrm{O}^{\prime}$.

(d) Termination: return $<\mathrm{A}$, TOLO $>$.

RTOP(<A, O, L $>$, agenda, Э) 
(1) Termination: if agenda is empty, return $<\mathrm{A}$, TOLO $>$.

(2) Goal selection: let $<\mathrm{Q}, \mathrm{A}_{\text {need }}>$ be a pair on the agenda (by definition $\mathrm{A}_{\text {need }} \in \mathrm{A}$ and $\mathrm{Q}$ is a precondition of $\mathrm{A}_{\text {need }}$ ).

(3) Operator selection: Let $\mathrm{A}_{\text {add }}$ be the action of choosing an operator that has $\mathrm{Q}$ as its one postcondition (an operator can be chosen from $\vartheta$, or an operator already in A that can be consistently ordered prior to $\mathrm{A}_{\text {need }}$ ). The choice of an operator can be implemented in such as way that the chosen operator will achieve maximum number of goals in agenda.

Let $\mathrm{L}^{\prime}=\mathrm{L} \cup\left\{\mathrm{A}_{\text {add }} \rightarrow(\mathrm{Q}) \mathrm{A}_{\text {need }}\right\}$, and Let $\mathrm{O}^{\prime}=\mathrm{O} \cup\left\{\mathrm{A}_{\text {add }}<\mathrm{A}_{\text {need }}\right\}$.

If $A_{\text {add }}$ is newly instantiated, then

$$
\begin{aligned}
& \mathrm{A}^{\prime}=\mathrm{A} \cup\left\{\mathrm{A}_{\text {add }}\right\} \\
& \left(\text { otherwise let } \mathrm{A}^{\prime}=\mathrm{A}\right) .
\end{aligned}
$$

If no such operator exists, then return failure. For each condition, keep all other choices as backtrack points.

(4) Update goal set: Let agenda' $=$ agenda $-\left\{<\mathrm{Q}, \mathrm{A}_{\text {need }}>\right\}$. If $\mathrm{A}_{\text {add }}$ is newly instantiated, then for each conjunct, $\mathrm{Q}_{\mathrm{i}}$, of its precondition add $<\mathrm{Q}_{\mathrm{i}}, \mathrm{A}_{\text {add }}>$ to agenda'.

(5) Causal link protection: For every operator $A_{\text {add }}$ that might threaten a causal link $A_{p} \rightarrow(S) A_{c} \in L^{\prime}$ choose a consistent ordering constraint, either

- Demotion: add $\mathrm{A}_{\text {add }}<\mathrm{A}_{\mathrm{p}}$ to $\mathrm{O}^{\prime}$, or

- Promotion: add $\mathrm{A}_{\mathrm{c}}<\mathrm{A}_{\text {add }}$ to $\mathrm{O}^{\prime}$.

If neither constraint is consistent, then return failure.

(6) Recursive invocation: $\operatorname{RTOP}\left(<A^{\prime}, O^{\prime}, L^{\prime}>\right.$, agenda', ७).

Fig. 2. Regressive generic total order planner, RTOPKLT

In the above algorithm, there are four parameters. The InitialState is the starting place where the planner begins to work. The Goal is the final state. The parameters KnowledgeletName and DomainName are used by the planner to search the existing knowledgelets. RTOPKLT starts with a search of knowledgelet based on the knowledgelet name or the domain name. Based on the search result, it either returns a plan immediately or invokes the subroutine RTOP. The return type is a two-tuple data structure. " $\mathrm{A}$ " is a set of operators included in the executable plan and TOLO is the total order constraint on "A". The total ordering constraints, TOLO, is guaranteed to be an action sequence that solves the planning problem [5].

The first parameter to subroutine RTOP is a three-tuple data structure: A is the chosen operator for the partial plan, $\mathrm{O}$ is the set of ordering constraints, and $\mathrm{L}$ is the set of causal link constraints. The second parameter is an agenda of goals that need to be supported by links. Each item on the agenda is represented as a pair $<Q, A_{i}>$ where $Q$ is a precondition or a conjunct of the precondition of $A_{i}$. The third parameter is $\vartheta$, which is the pool of operators selected by a knowledgelet. To achieve simplicity, we introduce two dummy operators: $A_{0}$ and $A_{\infty}$. $A_{0}$, the start operator, has no precondition and has the initial state as its effect; $A_{\infty}$, the end operator, has no effect and has the goal as its precondition. Subroutine RTOP always starts with a null plan: $A=\left\{A_{0}, A_{\infty}\right\}$, ordering constraint $O=\left\{A_{0}<A_{\infty}\right\}$, and causal link $L=\{\}$. Then, 
RTOP makes non-deterministic choices of operators until all the conjuncts of every operator's precondition have been supported by causal links and all the threatened links have been protected from possible interferences. In the rest of the paper, we use " " to mean a condition is not true.

\subsection{Example of Applying the RTOPKLT}

Let's use an example to illustrate how the RTOPKLT planner works. We use the following simple but illustrative planning problem. The following is the problem statement:

\section{Example 1 (block world)}

This problem involves a robot operating in a closed room. There are three blocks of equal size labeled as $A, B$, and $C$. There is a table that supports blocks. The robot may be commanded to move blocks one at a time, placing them on the table or on top of other blocks. Blocks may only be stacked one atop the other and the robot can easily reach all of the blocks. Suppose the exact position of the blocks on the table are not important and that there is enough room for all the blocks to be resting on the table at the same time. The initial state is $A$ and $B$ are on the table; $C$ is on top of $A$. The goal state is $A$ on top of $B ; B$ is on top of $C$; and $C$ is on table.

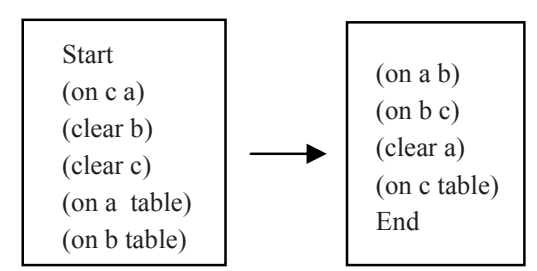

3.a. the null plan

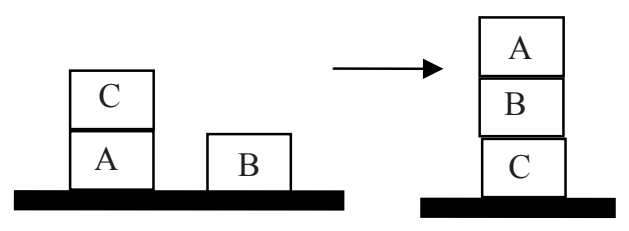

3.b. the initial state and the goal state

Fig. 3. The null plan, initial state, and goal state for block world example

Figure 3.b is the visual representation of the initial state and the goal state. The RTOPKLT starts by constructing a null plan as shown in figure 3.a.

\subsection{Solution to the Problem When There Is a Partial Plan}

If there is a solution existing in the applicable knowledgelet, then the RTOPKLT will return a solution immediately. The line (a) will return a plan of two-tuple < $\mathrm{A}, \mathrm{TOLO}>$. One possible answer is: the operator set A contains \{move-C-from-A-toTable, move-B-from-Table-to-C, move-A-from-Table-to-B $\}$, and TOLO contains \{move-C-from-A-to-Table $<$ move-B-from-Table-to-C $<$ move-A-from-Tableto-B\}. 


\subsection{Solution to the Problem When There Is No Partial Plan}

If there is no solution existing in the applicable knowledgelet, then the RTOPKLT will build a plan from scratch by calling the subroutine RTOP. When making the initial call, RTOPKLT passes in a null plan (shown in the iteration 1 of Table 1) as parameters. The parameters are: $\langle\mathrm{A}, \mathrm{O}, \mathrm{L}\rangle$, agenda, $\vartheta$. The values of each variable in a null plan will be the following: $A=\left\{A_{0}, A_{\infty}\right\}, O=\left\{A_{0}<A_{\infty}\right\}, L=\{\}$, agenda $=$ $\left\{<\right.$ (on A B ), $\mathrm{A}_{\infty}>,<$ (on B C), $\mathrm{A}_{\infty}>,<$ (on C Table), $\left.\mathrm{A}_{\infty}>\right\}$, and the operator pool $\vartheta$ that contains the relevant set shown in the first part of Table 1. Rest of Table 1 shows the computation results for each iteration of applying RTOPKLT to the example 1.

Table 1. The values of variables for each iteration

\begin{tabular}{|c|c|c|c|c|}
\hline $\begin{array}{l}\text { Operator } \\
\text { pool } \vartheta\end{array}$ & \multicolumn{4}{|c|}{ 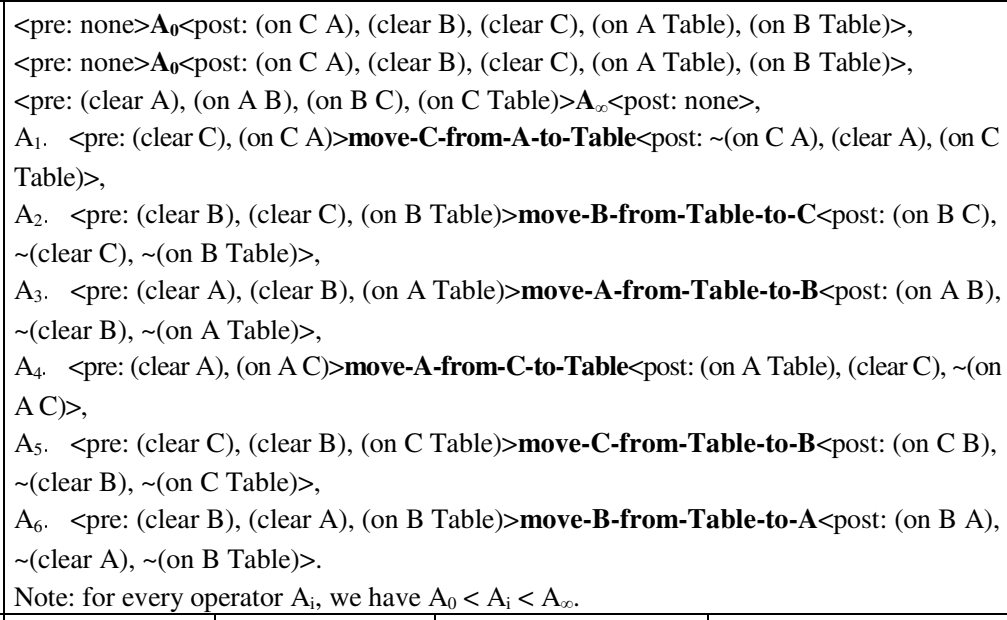 } \\
\hline Iteration & A & 0 & $\mathbf{L}$ & Agenda \\
\hline 1 & $\mathrm{~A}_{0}, \mathrm{~A}_{\infty}$ & $\mathrm{A}_{0}<\mathrm{A}_{\infty}$ & none & $\begin{array}{l}<(\text { clear } \mathrm{A}), \mathrm{A}_{\infty}>,<(\text { on } \mathrm{A} \\
\mathrm{B}), \mathrm{A}_{\infty}>,<(\text { on B C }), \mathrm{A}_{\infty}>\text {, } \\
<(\text { on C Table }), \mathrm{A}_{\infty}>\end{array}$ \\
\hline 2 & $\begin{array}{l}\mathrm{A}_{0}, \mathrm{~A}_{\infty} \\
\mathrm{A}_{2}\end{array}$ & $\begin{array}{l}\mathrm{A}_{0}<\mathrm{A}_{\infty} \\
\mathrm{A}_{0}<\mathrm{A}_{2} \\
\mathrm{~A}_{2}<\mathrm{A}_{\infty}\end{array}$ & $\begin{array}{l}\mathrm{A}_{2} \rightarrow\left(\text { on B C) } \mathrm{A}_{\infty}\right. \\
\mathrm{A}_{0} \rightarrow(\text { clear B }) \mathrm{A}_{2} \\
\mathrm{~A}_{0} \rightarrow\left(\text { clear C) } \mathrm{A}_{2}\right. \\
\mathrm{A}_{0} \rightarrow(\text { on B Table }) \mathrm{A}_{2}\end{array}$ & $\begin{array}{l}<(\text { clear } \mathrm{A}), \mathrm{A}_{\infty}>,<(\text { on } \mathrm{A} \\
\mathrm{B}), \mathrm{A}_{\infty}>,<(\text { on C Table }) \\
\mathrm{A}_{\infty}>\end{array}$ \\
\hline 3 & $\begin{array}{l}\mathrm{A}_{0}, \mathrm{~A}_{\infty} \\
\mathrm{A}_{2}, \mathrm{~A}_{3}\end{array}$ & $\begin{array}{l}\mathrm{A}_{0}<\mathrm{A}_{\infty} \\
\mathrm{A}_{0}<\mathrm{A}_{2} \\
\mathrm{~A}_{2}<\mathrm{A}_{\infty} \\
\mathrm{A}_{0}<\mathrm{A}_{3} \\
\mathrm{~A}_{3}<\mathrm{A}_{\infty}, \mathrm{A}_{2}<\mathrm{A}_{3}\end{array}$ & $\begin{array}{l}\mathrm{A}_{2} \rightarrow\left(\text { on B C) } \mathrm{A}_{\infty}\right. \\
\mathrm{A}_{0} \rightarrow(\text { clear B }) \mathrm{A}_{2} \\
\mathrm{~A}_{0} \rightarrow\left(\text { clear C) } \mathrm{A}_{2}\right. \\
\mathrm{A}_{0} \rightarrow(\text { on B Table }) \mathrm{A}_{2} \\
\mathrm{~A}_{3} \rightarrow(\text { on A B }) \mathrm{A}_{\infty}\end{array}$ & $\begin{array}{l}<(\text { clear } \mathrm{A}), \mathrm{A}_{\infty}>,<(\text { on } \mathrm{C} \\
\text { Table }), \mathrm{A}_{\infty}>, \\
<(\text { clear } \mathrm{A}), \mathrm{A}_{3}>,<(\text { clear } \\
\mathrm{B}), \mathrm{A}_{3}>,<(\text { on A Table }), \\
\mathrm{A}_{3}>\end{array}$ \\
\hline 4 & $\begin{array}{l}\mathrm{A}_{0}, \mathrm{~A}_{\infty} \\
\mathrm{A}_{2}, \mathrm{~A}_{3}\end{array}$ & $\begin{array}{l}\mathrm{A}_{0}<\mathrm{A}_{\infty} \\
\mathrm{A}_{0}<\mathrm{A}_{2} \\
\mathrm{~A}_{2}<\mathrm{A}_{\infty} \\
\mathrm{A}_{0}<\mathrm{A}_{3} \\
\mathrm{~A}_{3}<\mathrm{A}_{\infty} \\
\mathrm{A}_{2}<\mathrm{A}_{3}\end{array}$ & $\begin{array}{l}\mathrm{A}_{2} \rightarrow\left(\text { on B C) } \mathrm{A}_{\infty}\right. \\
\mathrm{A}_{0} \rightarrow(\text { clear B }) \mathrm{A}_{2} \\
\mathrm{~A}_{0} \rightarrow\left(\text { clear C) } \mathrm{A}_{2}\right. \\
\mathrm{A}_{0} \rightarrow(\text { on B Table }) \mathrm{A}_{2} \\
\mathrm{~A}_{3} \rightarrow(\text { on A B }) \mathrm{A}_{\infty} \\
\mathrm{A}_{0} \rightarrow(\text { clear B }) \mathrm{A}_{3} \\
\mathrm{~A}_{0} \rightarrow(\text { on A Table }) \mathrm{A}_{3}\end{array}$ & $\begin{array}{l}<(\text { clear } \mathrm{A}), \mathrm{A}_{\infty}>,<(\text { on } \mathrm{C} \\
\text { Table }), \mathrm{A}_{\infty}> \\
<(\text { clear } \mathrm{A}), \mathrm{A}_{3}>\end{array}$ \\
\hline
\end{tabular}


Table 1. (Continued)

\begin{tabular}{|c|c|c|c|}
\hline 5 & $\begin{array}{l}\mathrm{A}_{0}, \mathrm{~A}_{\infty}, \\
\mathrm{A}_{2}, \mathrm{~A}_{3} \\
\mathrm{~A}_{1}\end{array}$ & $\begin{array}{l}\mathrm{A}_{0}<\mathrm{A}_{\infty} \\
\mathrm{A}_{0}<\mathrm{A}_{2} \\
\mathrm{~A}_{2}<\mathrm{A}_{\infty} \\
\mathrm{A}_{0}<\mathrm{A}_{3} \\
\mathrm{~A}_{3}<\mathrm{A}_{\infty} \\
\mathrm{A}_{2}<\mathrm{A}_{3} \\
\mathrm{~A}_{0}<\mathrm{A}_{1} \\
\mathrm{~A}_{1}<\mathrm{A}_{\infty} \\
\mathrm{A}_{1}<\mathrm{A}_{2}\end{array}$ & $\begin{array}{l}\mathrm{A}_{2} \rightarrow\left(\text { on B C) } \mathrm{A}_{\infty}\right. \\
\mathrm{A}_{0} \rightarrow(\text { clear B }) \mathrm{A}_{2} \\
\mathrm{~A}_{0} \rightarrow\left(\text { clear C) } \mathrm{A}_{2}\right. \\
\mathrm{A}_{0} \rightarrow(\text { on B Table }) \mathrm{A}_{2} \\
\mathrm{~A}_{3} \rightarrow\left(\text { on A B) } \mathrm{A}_{\infty}\right. \\
\mathrm{A}_{0} \rightarrow \text { (clear B) } \mathrm{A}_{3} \\
\left.\mathrm{~A}_{0} \rightarrow \text { (on A Table }\right) \mathrm{A}_{3} \\
\mathrm{~A}_{0} \rightarrow \text { (clear C) } \mathrm{A}_{1} \\
\mathrm{~A}_{0} \rightarrow \text { (on C A) } \mathrm{A}_{1} \\
\mathrm{~A}_{1} \rightarrow \text { (clear A) } \mathrm{A}_{\infty} \\
\mathrm{A}_{1} \rightarrow \text { (clear A) } \mathrm{A}_{3} \\
\left.\mathrm{~A}_{1} \rightarrow \text { (on C Table }\right) \mathrm{A}_{\infty}\end{array}$ \\
\hline 6 & $\begin{array}{l}\mathrm{A}_{0}, \mathrm{~A}_{\infty}, \mathrm{A}_{2}, \mathrm{~A}_{3}, \\
\mathrm{~A}_{1}\end{array}$ & $\begin{array}{l}\mathrm{A}_{0} \ll \mathrm{A}_{1} \ll \mathrm{A}_{2} \\
\ll<\mathrm{A}_{3} \ll<\mathrm{A}_{\infty}\end{array}$ & \\
\hline
\end{tabular}

Initially, the planner contains only null plan as shown in iteration 1 of the above table. At this point, the agenda is: $<$ (clear $\mathrm{A}), \mathrm{A}_{\infty}>,<($ on $\mathrm{A} \mathrm{B}), \mathrm{A}_{\infty}>,<($ on $\mathrm{B} \mathrm{C}), \mathrm{A}_{\infty}>$, $<$ (on $\mathrm{C}$ Table), $\mathrm{A}_{\infty}>$. There are four choices for the immediate goal. As far as completeness is concerned, it doesn't matter which one is chosen first. If the first conditions take from agenda is (on B C) and the operator to support this condition, $\mathrm{A}_{\mathrm{add}}$, is move-B-from-Table-to-C, then all the values of parameters will be updated to the values as shown in iteration 2. $\mathrm{A}_{\text {add }}$ has three preconditions, they can either be supported by conditions in the initial state or be put into agenda. In this instance, all the preconditions are supported by the initial condition. We record this fact by adding three causal links to L.

For iteration 3, suppose the planner selects the goal (on A B) from the agenda and decides to instantiate a new operator 3 , move-A-from-Table-to-B, as $A_{\text {add }}$. If operator 3 is put before operator 2 , we will get a threat situation. We resolve threat by promotion to order the threat after link's consumer. In iteration 6 , the planner returns a totally ordered plan with two-tuples: $<A=\left\{A_{0}, A_{1}, A_{2}, A_{3}, A_{\infty}\right\}, T O L O=$ $\left\{\mathrm{A}_{0}<<\mathrm{A}_{1}<<\mathrm{A}_{2}<<\mathrm{A}_{3}<<\mathrm{A}_{\infty}\right\}>$. The total order uses notation " $<<$ " to indicate the absolute order among chosen operators. In this iteration, the agenda is empty and the algorithm terminates.

\section{References}

1. McDermott, D.: The 1998 AI planning systems competition, AI Magazine 21 (2) (2000) 35-55.

2. Bertino, E., Provetti, A., and Salvetti, F.: "Local Closed-World Assumptions for Reasoning about Semantic Web Data", In Proceedings of the APPIA-GULP-PRODE Conference on Declarative Programming (AGP '03), pages 314-323, 2003. 
3. Gordon, A.: "The Representation of Planning Strategies", Artificial Intelligence 153 (1-2) (2004) 287-305.

4. Akman, V., Erdogan, S., Lee, J., Lifschitz, V., Turner, H.: "Representing the Zoo World and the Traffic World in the Language of the Causal Calculator", Artificial Intelligence 153 (1-2) (2004) 105-140.

5. Wilkins, D.: Practical Planning Extending the Classical AI Planning Paradigm, Morgan Kaufmann, San Mateo, California, 1988.

6. Gray, R.: "Agent Tcl: A flexible and secure mobile-agent system," In Mark Diekhans and Mark Roseman, editors, Proceedings of the Fourth Annual Tcl/Tk Workshop (TCL Ô96), Monterey, California, July 1996. 